\title{
An Implementation of a Spike-Response Model With Escape Noise Using an Avalanche Diode
}

\author{
Thomas Clayton, Katherine Cameron, Member, IEEE, Bruce R. Rae, Member, IEEE, Nancy Sabatier, \\ Edoardo Charbon, Senior Member, IEEE, Robert K. Henderson, Member, IEEE, Gareth Leng, and \\ Alan Murray, Fellow, IEEE
}

\begin{abstract}
This paper introduces a novel probabilistic spike-response model through the combination of avalanche diode-generated Poisson distributed noise, and a standard exponential decay-based spike-response curve. The noise source, which is derived from a $0.35-\mu \mathrm{m}$ single-photon avalanche diode (kept in the dark), was tested experimentally to verify its characteristics, before being combined with a field-programmable gate-array implementation of a spike-response model. This simple model was then analyzed, and shown to reproduce seven of eight behaviors recorded during an extensive study of the ventral medial hypothalamic (VMH) region of the brain. It is thought that many of the cell types found within the VMH are fed from a tonic noise synaptic input, where the patterns generated are a product of their spike response and not their interconnection. This paper shows how this tonic noise source can be modelled, and due to the independent nature of the noise sources, provides an avenue for the exploration of networks of noise-fueled neurons, which play a significant role in pattern generation within the brain.
\end{abstract}

Index Terms-Modelling, single-photon avalanche diode (SPAD), spike-response model, spiking neuron.

\section{INTRODUCTION}

$\mathbf{T}$ HIS paper presents the initial proof of concept for a novel implementation of a probabilistic cumulative spike-response neural model (CSRM) with escape noise through the use of an avalanching diode acting as a Poisson distributed noise source. Most integrated-circuit (IC)-based spiking neural models are deterministic in nature, where incoming synaptic events are integrated on a capacitive membrane with either a firing threshold (integrate-and-fire-based models) [1], [2] or voltage-dependent feedback mechanisms (conductance-based models) [3]-[5] to determine spike generation. Deterministic models have been used extensively to investigate single-neuron

Manuscript received February 16, 2010; revised May 25, 2010 and September 22, 2010; accepted November 22, 2010. Date of publication January 28, 2011; date of current version May 25, 2011. The work was supported in part by EPSRC Grant EP/C516583/1 and in part by the Scottish Funding Council for the Joint Research Institute with the Heriot-Watt University, which is part of the Edinburgh Research Partnership in Engineering and Mathematics (ERPem). This paper was recommended by Associate Editor R. Etienne-Cummings.

T. Clayton, K. Cameron, B. R. Rae, R. K. Henderson, and A. Murray are with the Institute of Integrated Micro and Nano Systems, Joint Research Institute for Integrated Systems, School of Engineering, University of Edinburgh, Edinburgh, EH9 3JL, U.K. (e-mail: T.Clayton@ed.ac.uk; K.Cameron@ed.ac.uk).

N. Sabatier and G. Leng are with the Centre for Integrative Physiology, School of Biomedical Sciences, University of Edinburgh, Edinburgh, EH8 9XD, U.K.

E. Charbon is with TU Delft, Delft 2628 CD, The Netherlands.

Color versions of one or more of the figures in this paper are available online at http://ieeexplore.ieee.org.

Digital Object Identifier 10.1109/TBCAS.2010.2100392

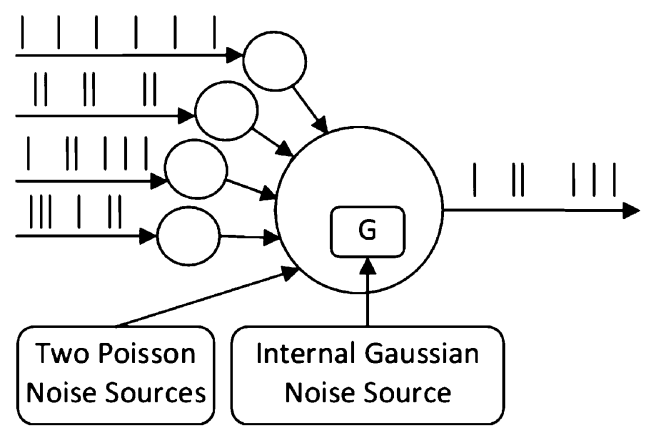

Fig. 1. Single-cell probabilistic neuron. Either two Poisson-distributed noise sources, or a single Gaussian, are added to represent synaptic noise originating outside the local network.

behaviors, and have even been incorporated with in-vitro experimentation to closely mimic the behavior of real neurons in real time [6]. Deterministic neural-network models have also been implemented extensively, but are nearly always sparsely connected due to the cost in chip area that large numbers of synapses represent [7]. Because of this, investigators tend to opt for implementations that utilize off-the-shelf memory architectures (such as those found in PCs and firmware, for example. FPGAs, graphics-processing units (GPUs), or super computers), which have more efficient memory structures to hold synaptic weight data and, therefore, allow larger networks with more synapses. However, even with efficiency gains through their use, network models still tend to be constructed with at least one order of magnitude fewer interconnections than the biology [8]-[10]. This leads to the need to simplify the topology to a sparsely connected network. See [11] for examples of possible population dynamics. One of these simplifications involves representing the local network behavior with a sparsely connected network model combined with a noise source at each neuron. The noise source represents the combined effects of synaptic stimulus originating from outside the network (Fig. 1). Although this is a broad simplification, it is thought that the synaptic inputs for many of the neurons within the hypothalamus can be represented by two Poisson distributed noise sources outside the cell, or a Gaussian noise source within [12]. As a further simplification, the cell membrane and firing mechanism can be replaced by a Poisson-distributed noise source, with the local activity modulating its mean rate instead of being integrated onto the cell membrane. As with the integrate and fire model, this method requires the enforcement of a refractory period to reduce the probability of firing directly 
after a spike event and, hence, remove events that correspond to small interspike intervals.

Noise is widely used with neural networks. Within probabilistic structures, such as the Boltzmann machine [13], noise can be used to escape local minimas and represent the natural variability of the data. In spiking neural networks, it has been observed that the mutual information transfer can be increased by stochastic resonance [14]. In addition, Fusi [15] showed that if stochastic learning takes place, the length of time that memories can be stored increases.

Creating a noise source with the required characteristics is not straightforward. A review of techniques for creating noise sources can be found in [16]; however, few are suitable for very-large scale-integrated (VLSI) implementation. Alspector et al. [17] required a noise source for a modified Boltzmann machine VLSI implementation but found that although amplifying the thermal noise of a resistor was a good source of noise, highfrequency oscillations caused the noise generators to correlate. They developed an alternative technique to produce Gaussian noise that did not suffer from correlations, and was suitable for VLSI implementation [18], but it was generated using a linear feedback shift register and was therefore only pseudorandom. If spiking noise is required, Chicca and Fusi showed how a randomly connected recurrent network can be used to generate a stochastic spike train [19].

This paper focuses on the development and testing of a probabilistic cumulative spike response model with escape noise, which utilizes the dark count of a single-photon avalanche diode (SPAD) to represent the cell membrane and firing mechanism. This paper shows that the dark count of a SPAD, when filtered for trapped charge effects, can be considered a pure Poisson distributed noise source. Furthermore, multiple SPADs used in this manner produce uncorrelated events, even when located next to each other on the substrate. Finally, we show that many of the single neuron behaviors recorded from the hypothalamic region of the brain can be replicated by applying a cumulative spike response mechanism to modulate the probability of avalanche diode-generated events. Replicated behaviors include spike adaptation, bursting, doublet, random, and regular activity.

The remainder of this paper is organized as follows. Section II introduces the cumulative spike-response model, highlighting its ties to observed biological phenomena. Section III introduces the SPAD architecture, discusses dark count, and why it is a suitable noise source, before presenting the method and results of experimentation to determine the quality of the noise produced. Section IV presents the proof-of-concept combination of the cumulative spike response (CSR) mechanism and SPAD noise source to create an avalanche diode-fuelled neural model. The method of implementation and results are presented, and the limitations of this form of model are discussed. This paper is drawn to a close by a brief conclusion.

\section{INTRODUCTION TO SPIKE-RESPONSE MODELS}

The spike-response model (SRM) [20]-[24] is an extension of the leaky integrate-and-fire (IF) model, where the membrane potential of the cell is derived from the time since the last action potential initiation, and the time since each incoming synaptic

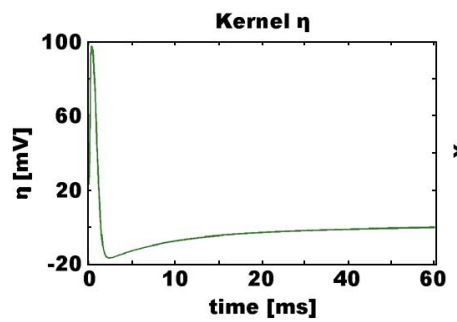

(a)

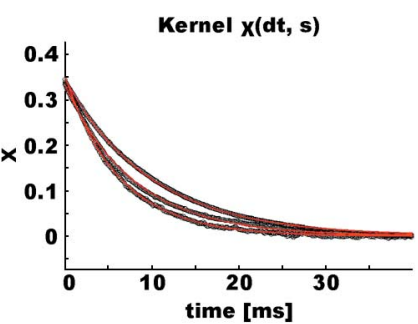

(b)
Fig. 2. Graphs showing the kernels that control the model's response over time to (a) an outgoing action-potential and (b) three incoming synaptic events. The outgoing action-potential consists of an initial peak (the spike) followed by a refractory period which greatly decreases the probability of refiring. The incoming synaptic events are represented by a single exponential decay (the red lines are a best fit to different values of this exponential). (Both images are reprinted from [24].)

event. The impact of the cells' own action potential and incoming synaptic events is defined by two kernels that describe the shape of their impact with time. In this way, the SRM introduces a measure of refractoriness to the IF model that describes a combination of effects, such as increasing threshold, hyperpolarizing afterpotential, and post-action-potential reduced responsiveness

$$
u(t)=\eta(t-\hat{t})+\int_{0}^{\infty} \kappa(t-\hat{t}, s) I(t-s) d s .
$$

Equation (1) describes the membrane potential as a function of time $(t)$. The neuron fires when the membrane voltage crosses the dynamic threshold, also enforced by a kernel $\theta(t-\hat{t})$, where $\hat{t}$ is the firing time of the last spike. It is constructed through the sum of the influence from the kernel that describes the shape of the membrane potential, both during and postfiring $(\eta)$, and the response $(\kappa)$ to the influence from each synaptic input $I(t-s)$. An example of the kernels $\kappa$ and $\eta$ is shown in Fig. 2.

The advantage of this form of model is that it can be made to produce a biologically realistic response to incoming synaptic events, while still being extremely simple in structure. This makes the SRM an ideal candidate for network models [25] as well as being easily adaptable to experimental data [26]. However, the basic SRM cannot represent slow activity-dependent effects, of the order of seconds, which are involved in slow bursting (such as the phasic firing patterns of vasopressin cells [27]). The cumulative spike-response model (CSRM) [28] provides the solution to this problem. Where the SRM is only interested in the most recent action potential, the CSRM calculates its membrane potential from the combined effects of several spikes, with synaptic events treated in the same manner as within the original SRM (2). $t^{k}$ is the times of all previous firings

$$
u(t)=\sum_{t^{k}} \eta(t-\hat{t})+\int_{0}^{\infty} \kappa(t-\hat{t}, s) I(t-s) d s .
$$

Both the adaptive exponential model [29] and Izhikevich's model [30], include cumulative adaptive elements within them. However, these models were not used as only the spike-response 
elements, and not a firing mechanism, were required. In addition, as described later in Section II-B, two adaptive variables (DAP and AHP) were essential for the reproduction of experimental VMH data, as opposed to the one (AHP) present in both of the aforementioned models.

\section{A. Noise and the SRM}

The SRM and CSRM can be simplified by replacing the synaptic input with a noise source, which then allows their study through the cell's probability of firing. This model, which has been called a stochastic threshold model [23], can now be analyzed analytically as a probability density function (PDF) of the model's ISI distribution. The same process can be performed for CSRMs but the calculation of the PDF is significantly more complex.

The most direct noise source implementation is to employ two Poisson-distributed sources in the place of all synapses, one to represent all excitatory presynaptic potentials (EPSPs), and the other to represent the inhibitory ones (IPSPs). These can be controlled to dictate the ratio (excitatory to inhibitory) of incoming events. Alternatively, if control of this ratio is not required, a Gaussian source may be instead implemented internally, as a diffusion approximation of many synaptic inputs. Finally, a Poisson-distributed source can be used as the output of the neural model. With this implementation, the probability of spike generation is modulated by the spike-response pattern to create the natural refractory periods produced from real neurons. This implementation, which can be considered to be an SRM with escape noise, has been chosen for our hardware implementation.

\section{B. Probabilistic CSRM}

The model presented in this paper is a CSRM with escape noise, using a series of summed exponential decays to represent the accumulation of all previous spike responses. Each exponential corresponds to an excitatory or inhibitory post-action-potential membrane dynamic (the hyperpolarizing after potential (HAP) [31], depolarizing after potential (DAP), and after hyperpolarization (AHP) [27], [32]). The HAP is a brief hyperpolarization (half life $-10 \mathrm{~s}$ of milliseconds) that sets the relative refractoriness of a cell after a spike-it can be modelled well with an SRM. The AHP is a much smaller and slower (half life —of the order of seconds) post-spike hyperpolarization that accumulates with successive spikes - this cannot be modelled with an SRM but instead requires a CSRM. The DAP characteristics fall between the two in magnitude and half life, and can be modelled by an SRM. However, longer term behaviors, such as phasic firing, require a CSRM since they can only be produced through the creation and suppression of a plateau of summed DAP activity. When combined with the noise-source control parameter, this creates a 7-D parameter space where pairs of parameters can be directly tied to the magnitude and duration of specific identifiable intrinsic cell characteristics.

This form of model is computationally simple because the model's spike response is a sum of exponentials, where each can be calculated at every time step by single multiplication. In addition, the response to the entire spike history, instead of just the most recent spikes, can be calculated at the same time using the same method. This is because the overall spike response over time is split by individual post-action-potential membrane dynamics, which are described as

$$
\frac{d M}{d t}=-\frac{M}{\tau_{M}}+M_{S T} \sum_{t^{S}<t} \delta\left(t-t^{S}\right)
$$

where $M$ is the magnitude of the post-synaptic action potential membrane dynamic, $\tau_{M}$ is the time constant of the membrane dynamic, the Dirac delta function $\delta$ approximates the effect of spike production upon the membrane dynamic through the addition of an $M_{S T}$-sized step increase or decrease, and $t^{S}$ is the time of spike production. With fixed control parameters for $\tau_{M}$ and $M_{S T}$, the bulk of the function can be simplified prior to simulation in discrete time to a single multiplication and a possible addition

$$
\Delta M=-\frac{M \Delta t}{\tau_{M}}+M_{\mathrm{ST}} \delta_{t, t^{s}}
$$

where $\Delta t$ is the size of the simulation time step, and $\delta_{t, t^{s}}$ is the Kronecker delta function which is equal to 1 when $t$ is equal to $t^{s}$.

The integration of balanced (50\% excitatory, 50\% inhibitory) incoming synaptic noise on the membrane, combined with a threshold firing mechanism, is represented by the probability of a Poisson-distributed noise source generating a spike. The spike-response pattern, generated by the summed exponential decays representing a neuron's HAP, DAP, and AHP, applies an offset to the mean firing rate of the noise source and, therefore, facilitates a refractory period in addition to the creation of more complex firing patterns. This is described as

$$
P(S)=f\left(\lambda_{\text {base }}+E_{\mathrm{HAP}}+E_{\mathrm{DAP}}+E_{\mathrm{AHP}}\right) \text { when } P(S) \ll 1
$$

where $P(S)$ is the probability of spike generation for a given time step. This is kept much smaller than 1 by reducing the size of the time step. The time step used was $1 \mathrm{~ms}$. Since most hypothalmic neurons fire at a rate much lower than $50 \mathrm{~Hz}, P(S)$ should have a base value of less than $0.05 . E_{\mathrm{HAP}}, E_{\mathrm{DAP}}$, and $E_{\mathrm{AHP}}$ represent the current value of the individual exponentials that form the spike response, and $f(\ldots)$ is a nonlinear transfer function that converts the spike response into a probability of firing. The spike response is first combined with the base probability of firing $\lambda_{\text {base }}$ before this function is applied. This function is explained in more detail in Section III since it is dependent on the Poisson-distributed random process.

The reason a Poisson-distributed noise source can be utilized in this fashion is due to the similarity between recorded cell data and a purely Poisson-distributed process. This can be shown by using the hazard function [33], [34], which is a modification of the ISI histogram, $I(n)$, where $n$ is the bin number of the ISI histogram. It is calculated as follows:

$$
H(n)=\frac{I(n)}{\sum_{n}^{\infty} I(n)} .
$$

The hazard function represents the probability of a spike event being generated at a given time interval since the previous event, provided that a subsequent event has not yet 

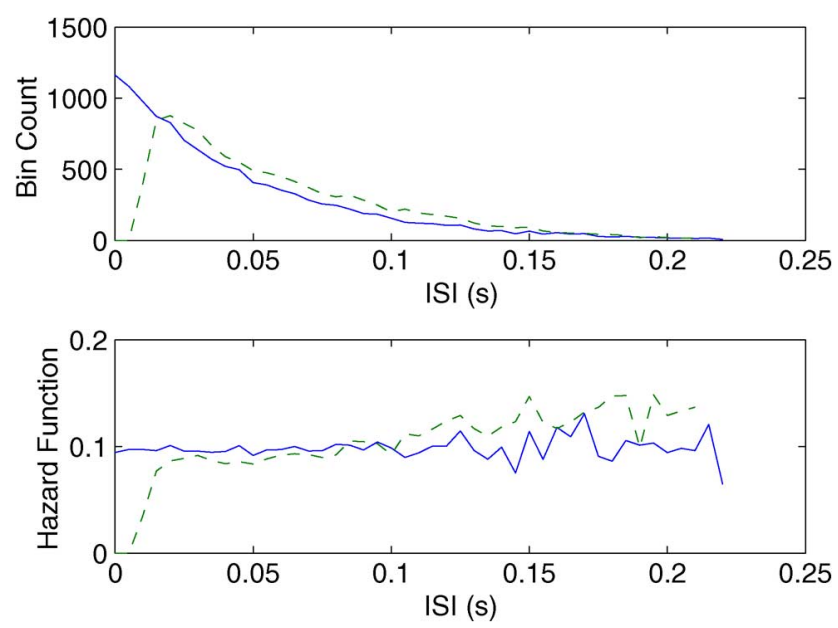

Fig. 3. Interspike interval (ISI) in five millisecond bins and hazard function of data recorded from a randomly firing VMH cell (dashed line) and a Matlabgenerated Poisson spike train (solid line).

occurred. A Poisson-distributed process has a constant probability of producing an event irrespective of the time since the previous event, which is shown as a flat distribution. Fig. 3 shows the hazard functions of a randomly firing neuron and a Poisson-distributed noise source generated in Matlab. The recorded neuronal pattern is similar except for a refractory period immediately after a spike event, which is enforced to protect the cell from death due to overactivity. This shows that after this initial refractory period, the statistics are consistent with a homogeneous Poisson process. Sabatier and Leng [35] performed a study of cell-firing patterns recorded from the $\mathrm{VMH}$, and showed that when normalized by their mean firing rate, they could be divided into nine distinct groups according to their hazard functions. These groups, with the exception of oscillatory behavior, are shown in Fig. 4. By altering the ratio of the magnitudes and half lives of the exponential decays that form the spike response, this paper shows that similar behaviors can be generated from this Poisson-driven probabilistic CSRM. No attempt was made to reproduce the oscillatory behavior since it is thought to be a product of network phenomena, and not intrinsic cell properties.

The bulk of the model complexity is tied to an easily conceptualized and computational simple membrane response to cell activation. Since the spike response is the sum of three exponentially decaying variables, a solution could be constructed using either digital or analog; however, for this proof of concept, a digital implementation within an FPGA was chosen due to the low development costs.

\section{Avalanche Diode-Driven Poisson-Distributed NOISE SOURCE}

\section{A. Introduction to SPADs}

The single-photon avalanche diode (SPAD) allows single-photon detection through the action of avalanche breakdown in a $p-n$ photodiode, reverse biased above its breakdown voltage (Geiger mode). In Geiger mode operation, the gain of a SPAD becomes virtually infinite and, with suitable biasing, a transistor-transistor logic (TTL)-compatible pulse is produced
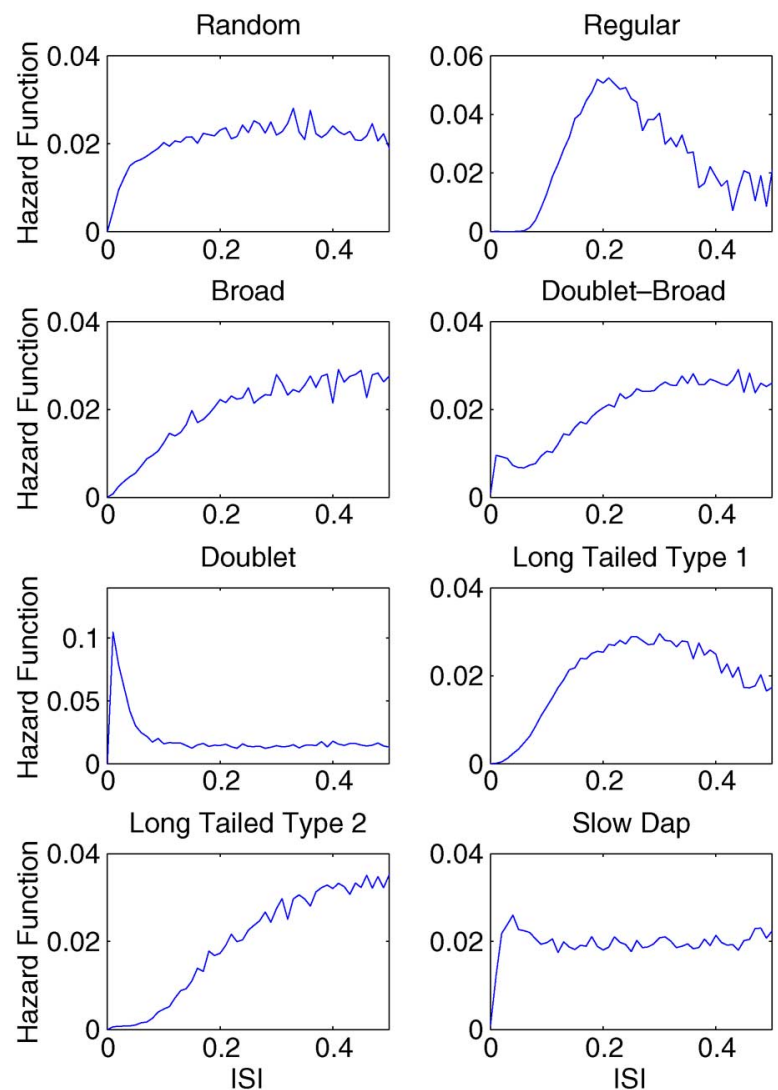

Fig. 4. Hazard function calculated from cell recordings during eight different types of behavior [35].

upon detection of a single photon. The concept of the avalanche photodiode was first proposed by Haitz et al. [36] and, in recent years, much work has focused on implementing single-photon avalanche diodes in a standard complementary metal-oxide semiconductor (CMOS) process [37]-[40]. SPADs realized in a foundry CMOS process were first demonstrated by Rochas et al. in 2002 [41].

A diode biased beyond its reverse bias breakdown voltage will remain in a quiescent state (zero current flowing) for a relatively long period of time (in the order of milliseconds). It is the occurrence of a primary-free carrier within the high electric field $p$ - $n$ junction that triggers an avalanche breakdown event [42]. When operated as a photodetector, it is always hoped that this primary-free carrier is generated as a result of an incident photon. However, spurious breakdown events do occur due to thermal- or tunnel-generated carriers and trapped charges.

If a free electron-hole pair is generated within the depletion region of the $p-n$ junction, the high electric field caused by the large reverse bias voltage will accelerate the electrons and holes toward the $p$ and $n$ regions, respectively. The accelerated free electron and hole collide with static electron-hole pairs in the junction, resulting in impact ionization. These newly created free electrons and holes are subsequently accelerated, resulting in further collisions and, hence, ionization events. As the number of free electron-hole pairs increases, the current flowing through the SPAD exponentially increases until quenching occurs. 


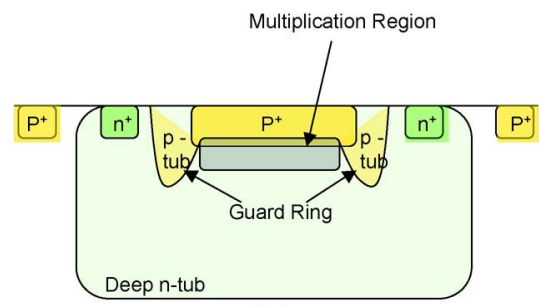

(a)

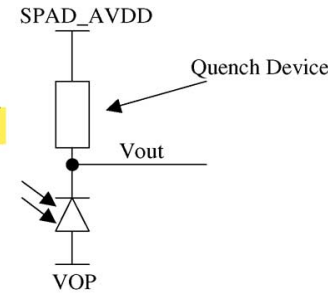

(b)
Fig. 5. (a) Structure of the SPAD device. (b) SPAD with quench resistor.

Dark counts are non-photon-induced breakdown events and are a function of detector area and temperature. The primary causes of dark counts are thermal- or tunnel-generated carriers in the diode $p$ - $n$ junction [42]. As such, the dark count rate (DCR) of a SPAD is strongly temperature dependent and follows the temperature dependence of these mechanisms. As a rule of thumb, the dark count doubles every $10^{\circ} \mathrm{C}$ [42]. Dark count is also dependent on the reverse bias voltage placed across the SPAD. As this voltage is increased, the SPADs sensitivity increases, due to the higher electric field at the $p$ - $n$ junction increasing the likelihood of an avalanche breakdown event. However, the increased probability of breakdown also increases the probability of a nonphoton-induced breakdown occurring. It is this method of electron-hole pair generation that will be used as the noise source.

After-pulsing is defined as spurious counts caused by carriers temporarily trapped in the depletion region during a breakdown event. After a short time, these charges are released, causing a secondary Geiger pulse. The level of after-pulsing in a device is dependent on the quality of the silicon (which defines the trap concentration) and the number of carriers generated during a breakdown event [43]. The number of carriers generated is dependent on the parasitic capacitance of the diode and the quench circuitry used. If the hold-off time of the quench circuit is not long enough, trapped charges do not dissipate and after-pulsing occurs. As quench time increases, the correlation between the initial pulse and after-pulses decreases. After-pulsing also shows an inverse temperature dependence, increasing as temperature decreases [44].

SPAD Structure and Biasing: The SPAD detector implemented in this project consists of a circular dual-junction structure: $p+$ anode/deep $n$-well $/ p$-substrate [Fig. 5(a)]. The $p+$ anode/deep $n$-well junction forms the avalanche multiplication region where the Geiger breakdown occurs. The $n$-well $/ p$-substrate junction allows the $p+$ anode to be biased independently from the substrate and prevents electrical crosstalk. A $p$-well guard-ring surrounds the $p+$ anode to prevent premature breakdown [45]. The device has a diameter of $6 \mu \mathrm{m}$, resulting in an active area of $28.27 \mu \mathrm{m}^{2}$.

To ensure the SPAD detector's compatibility with standard CMOS circuitry, the biasing of the SPAD must be carefully considered. The logic levels of the $0.35-\mu \mathrm{m}$ process used in this project were $0 \mathrm{~V}(\operatorname{logic} 0)$ and $3.3 \mathrm{~V}(\operatorname{logic} 1)$. Therefore, it had to be ensured that the output transition of the SPAD on breakdown had a 3.3-V swing. This is achieved by setting up appro- priate bias conditions. For the SPAD to operate in Geiger mode, it must be biased above its breakdown voltage. The $p+$ anode of the SPAD is therefore biased at a high negative voltage VOP [Fig. 5(b)]. The deep $n$-well cathode is connected to a positive power supply SPAD_AVDD, via a quench resistor.

The key is to ensure that the excess bias voltage across the SPAD when in an armed state is equal to $3.3 \mathrm{~V}$ (for a $0.35-\mu \mathrm{m}$ process). The excess bias voltage is defined as

$$
V e=\mathrm{VOP}+\mathrm{SPAD} \_\mathrm{AVDD}-V b d
$$

where $V e$ is the SPAD excess bias voltage, and $V b d$ is the reverse bias breakdown voltage of the diode. At the onset of Avalanche breakdown in the photodiode, current begins to flow in the device. As this current increases, the voltage dropping across the quench resistor increases, thus lowering the reverse bias voltage across the diode. This process continues until the diode is brought out of Geiger mode and the avalanche process is halted. According to (7), the transition required to bring the SPAD out of avalanche breakdown is equal to $V e$. As the current through the diode is reduced, the voltage dropping across the quench resistor is reduced. In turn, the photodiode reverse bias voltage increases until the diode returns to Geiger mode operation. The SPAD is now rearmed and ready to detect the next photon. The quench resistor is sized so that the voltage transition seen at the SPAD output [Vout, Fig. 5(b)] during this process is compatible with standard CMOS logic cells.

If the reverse bias across the SPAD is insufficient, the SPAD will never breakdown. Conversely, if the reverse bias voltage is too high, the SPAD will be in permanent breakdown. For the SPAD used in this project, the minimum reverse bias voltage was found to be approximately $20.8 \mathrm{~V}$. The SPAD went into permanent breakdown at voltages above approximately $24.05 \mathrm{~V}$.

\section{B. Experimental Setup}

To test the viability of avalanche diodes as a source of Poisson-distributed random events, an application-specific integrated circuit (ASIC), which included nine independently wired SPADs, was shielded from all light sources and analyzed. This analysis focused on how changes to the off-chip bias voltage affected the mean firing rate as well as determining if there was any cross correlation between adjacent SPADs. The correlations could be caused by optical or electrical crosstalk [45]. These types of crosstalk would both increase the likelihood of neighboring SPADs firing, but another type of correlation could be present. When an SPAD fires, quenching current is drawn from VEB since this is a global signal and any variation in it will affect the probability of firing all of the other SPADs. Any of these would be highly detrimental to any future neural-network implementation.

SPAD events were collected via an FPGA ${ }^{1}$ and time-stamped before being passed to custom software via USB for analysis. Each timestamp (24 b) was accompanied by an address ( $8 \mathrm{~b}$ ). The FPGA was clocked at $200 \mathrm{MHz}$, well above the Nyquist frequency for these particular SPADs (maximum output frequency of $30 \mathrm{MHz}$ ).

\footnotetext{
${ }^{1}$ The FPGA was on an Opal Kelly XEM 3010 Development Board.
} 

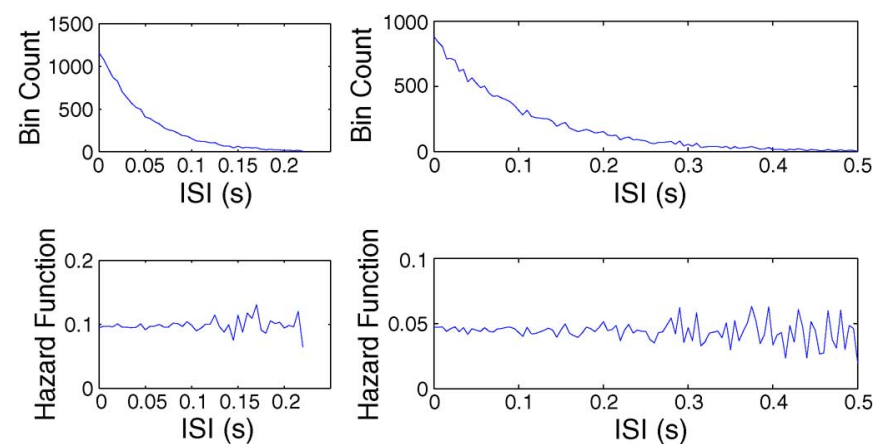

(a)

(b)

Fig. 6. Interspike interval (ISI), in five millisconds bins, and hazard function of (a) a Matlab-generated Poisson spike train, reproduced from Fig. 3 and (b) recorded dark count events from a single SPAD.

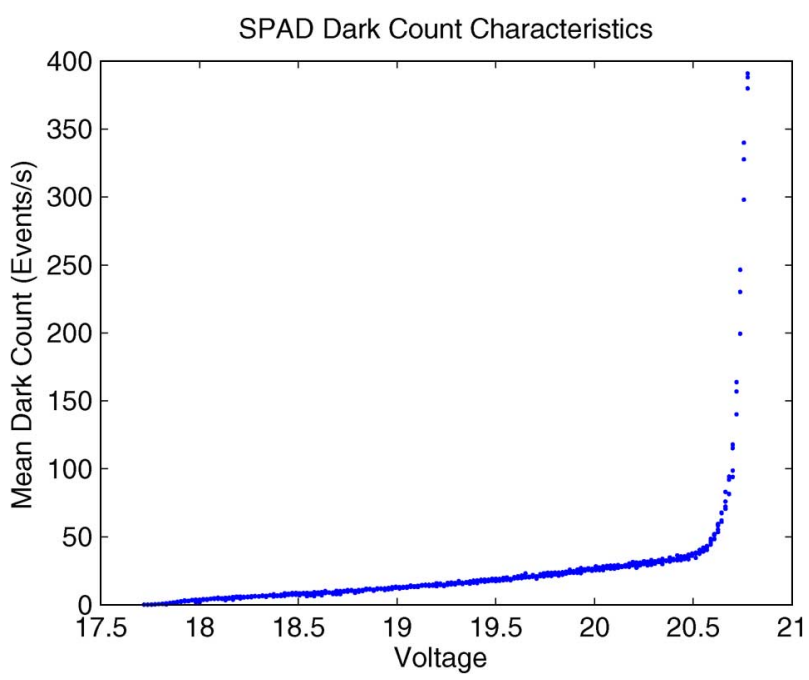

Fig. 7. Mean frequency of the dark count when VOP is altered. Below $20.5 \mathrm{~V}$, there is an approximately linear relationship between voltage and probability of firing.

\section{Results}

The first test undertaken was to look at the events recorded from a single SPAD and plot the ISI distribution and corresponding hazard function in order to verify that the SPAD-generated dark events were indeed Poisson distributed. The recorded events were first preprocessed to remove the spikes caused by after-pulsing. Any spike that occurred within $200 \mu \mathrm{s}$ of the previous event was deleted from the spike train. Fig. 6 shows the results and a comparison with Fig. 3 indicates that a SPAD can indeed produce a Poisson-distributed spike train. To further test the spike train, a negative exponential curve was fitted to the ISI histogram data, bin width $5 \mathrm{~ms}$, and the coefficient of variation (CV), defined as $\sigma(i s i) /$ mean $(i s i)$, was also calculated. The curve was fitted with an adjusted $\mathrm{R}^{2}$ value of 0.9966 and the CV was 1.0195 (a perfect Poisson spike train has a value of 1 for both). These results, when combined with the near flatness of the hazard function in Fig. 6(b), give confidence that the spike trains can be regarded as Poisson.

The mean frequency of the spike train can be altered by VOP - the large negative voltage applied to the SPAD. Tests were run to find the range of frequencies that could be achieved. The mean dark count was recorded by the FPGA for voltages ranging from $17.71 \mathrm{v}$ to $21 \mathrm{~V}$. The results are shown in Fig. 7. This mean dark count can be interpreted as the probability

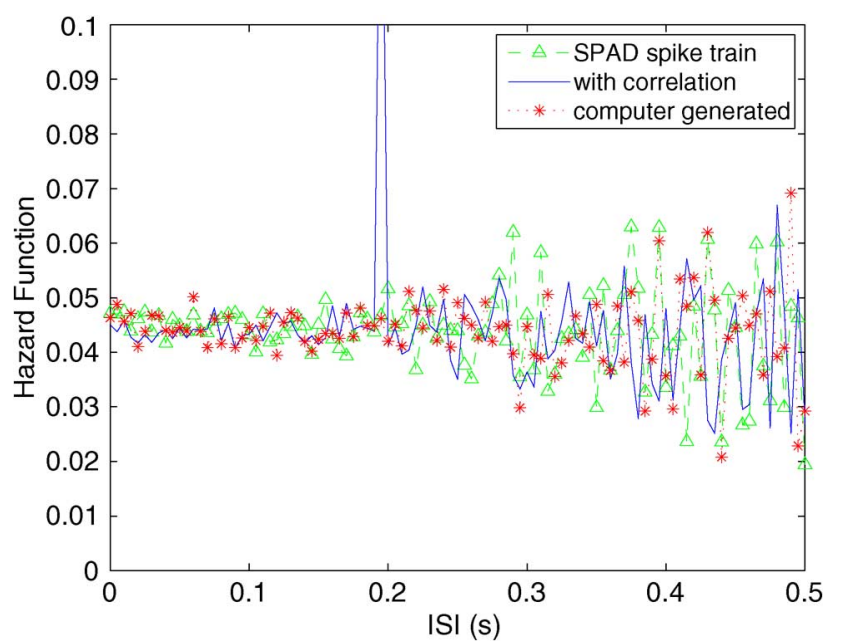

Fig. 8. Three hazard functions. The dashed line marked with triangles is the hazard function of a SPAD-generated spike train. The dotted line marked with asterisks is the hazard function calculated from the inter spike intervals between a Matlab-generated Poisson spike train and the spike train from the SPAD. The solid line is the hazard function between two spike trains with artificial correlations inserted between them. The increase in probability of firing $200 \mathrm{~ms}$ after an event on the previous spike train is clearly visible.

of firing given a varying VOP. This allows us to implement (5) with VOP representing $\lambda_{\text {base }}+E_{\mathrm{HAP}}+E_{\mathrm{DAP}}+E_{\mathrm{AHP}}$. Above $20.5 \mathrm{~V}$, the electric field across the SPAD becomes so high that the SPAD begins to break down laterally, in a more regular manner, and, therefore, the spike train loses its Poisson distribution. The results show that we can achieve a mean frequency of up to $40 \mathrm{~Hz}$ which is a good range for single neuron models. An array of 1024 of these SPADs has been reported in [46]. The event readout is address based, allowing the spike trains to be merged if a faster frequency is required.

It is also important to determine that the Poisson-distributed spike trains from neighboring SPADs are independent. If two Poisson-distributed spike trains are independent, the interspike interval between these two trains will also be Poisson. To illustrate this, three spike trains were generated: A) a recorded spike train from a SPAD that was preprocessed to remove the effects of after-pulsing. This was done by removing any spike that occurred within $200 \mu$ s of the preceeding pulse; B) A Matlab-generated Poisson spike train with a mean frequency of $15.5 \mathrm{~Hz}$; and C) a spike train recorded from another SPAD which had a $10 \%$ artifical correlation to A added to it. Fig. 8 shows three different hazard functions created from these spike trains. The line marked with triangles is the hazard function of spike train A. As expected, the hazard function is flat, indicating a Poisson-distributed spike train. The Matlab-generated Poisson spike train (B) was then compared to the train from the SPAD (A). The ISI from every spike in B to the next spike in A was calculated and the hazard function determined. This result is shown by the line marked with asterisks. Since the spike trains are independent, the hazard function is flat and has the same probability of firing as spike train A. Finally, the hazard function between trains $\mathrm{C}$ and $\mathrm{A}$ was calculated and is shown as the solid line in Fig. 8. The peak in the hazard function clearly indicates a correlation is present.

To test that the spike trains were not correlating, the same process was performed on data recorded from eight neighboring 
TABLE I

Adjusted $R^{2}$ VAlues After CuRve FitTing AND the CoEfFicient of VARiation (CV)

\begin{tabular}{|c|c|c|c|c|c|c|c|c|}
\hline Adj. $\mathrm{R}^{2}$ & \multicolumn{9}{|c|}{ ISI measured From Spike Train } \\
\hline To Spike Train & $\mathrm{A}$ & $\mathrm{B}$ & $\mathrm{C}$ & $\mathrm{D}$ & $\mathrm{E}$ & $\mathrm{F}$ & $\mathrm{G}$ & $\mathrm{H}$ \\
\hline A & $\mathbf{0 . 9 9 6 5}$ & 0.9963 & 0.9970 & 0.9951 & 0.9957 & 0.9958 & 0.9972 & 0.9965 \\
\hline B & 0.9966 & $\mathbf{0 . 9 1 6 6}$ & 0.9966 & 0.9930 & 0.9939 & 0.9945 & 0.9955 & 0.9963 \\
\hline C & 0.9948 & 0.9947 & $\mathbf{0 . 9 4 6 3}$ & 0.9951 & 0.9921 & 0.9944 & 0.9939 & 0.9975 \\
\hline D & 0.9941 & 0.9924 & 0.9942 & $\mathbf{0 . 9 2 3 0}$ & 0.9935 & 0.9932 & 0.9943 & 0.9947 \\
\hline E & 0.9953 & 0.9941 & 0.9958 & 0.9938 & $\mathbf{0 . 9 9 4 0}$ & 0.9946 & 0.9966 & 0.9959 \\
\hline F & 0.9955 & 0.9933 & 0.9951 & 0.9938 & 0.9952 & $\mathbf{0 . 9 9 4 4}$ & 0.9951 & 0.9943 \\
\hline G & 0.9975 & 0.9957 & 0.9970 & 0.9961 & 0.9958 & 0.9972 & $\mathbf{0 . 9 5 6 4}$ & 0.9961 \\
\hline H & 0.9970 & 0.9964 & 0.9966 & 0.9955 & 0.9970 & 0.9975 & 0.9974 & $\mathbf{0 . 9 9 5 4}$ \\
\hline
\end{tabular}

\begin{tabular}{|c|c|c|c|c|c|c|c|c|}
\hline CV & \multicolumn{9}{|c|}{ ISI measured From Spike Train } \\
\hline To Spike Train & $\mathrm{A}$ & $\mathrm{B}$ & $\mathrm{C}$ & $\mathrm{D}$ & $\mathrm{E}$ & $\mathrm{F}$ & $\mathrm{G}$ & $\mathrm{H}$ \\
\hline $\mathrm{A}$ & $\mathbf{1 . 0 1 9 4}$ & 1.0103 & 0.9972 & 1.0102 & 1.0090 & 1.0152 & 1.0135 & 1.0324 \\
\hline B & 0.9927 & $\mathbf{1 . 0 5 0 7}$ & 1.0147 & 0.9992 & 1.0005 & 0.9940 & 0.9889 & 0.9991 \\
\hline C & 0.9846 & 0.9950 & $\mathbf{1 . 0 2 5}$ & 0.9891 & 0.9948 & 0.9898 & 0.9823 & 0.9860 \\
\hline D & 0.9872 & 0.9837 & 0.9916 & $\mathbf{1 . 0 2 1 3}$ & 0.9970 & 0.9976 & 0.9940 & 0.9838 \\
\hline E & 0.9979 & 1.0105 & 1.0003 & 0.9992 & $\mathbf{1 . 0 2 6 5}$ & 0.9909 & 0.9994 & 0.9941 \\
\hline F & 0.9861 & 1.0012 & 0.9835 & 0.9790 & 0.9901 & $\mathbf{0 . 9 9 1 3}$ & 0.9874 & 0.9772 \\
\hline G & 0.9839 & 0.9928 & 0.9935 & 0.9997 & 0.9998 & 1.0114 & $\mathbf{1 . 0 3 0 8}$ & 1.0000 \\
\hline H & 0.9755 & 0.9961 & 0.9999 & 1.0068 & 0.9980 & 0.9879 & 1.0022 & $\mathbf{1 . 0 1 0 0}$ \\
\hline
\end{tabular}

A

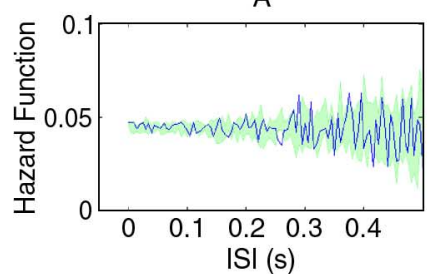

C

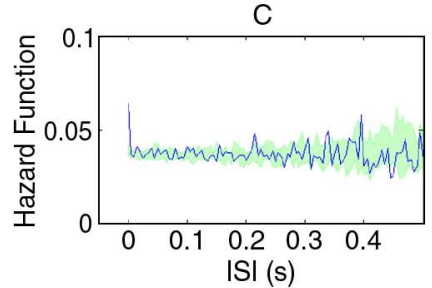

E

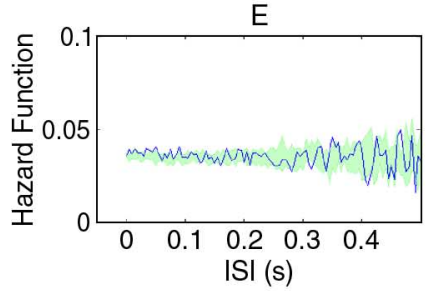

G

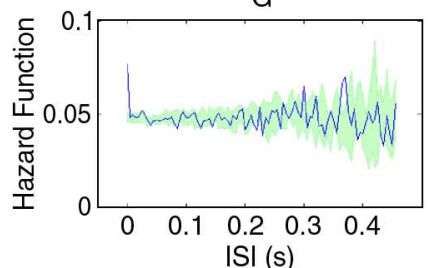

$|\mathrm{S}|(\mathrm{s})$
B

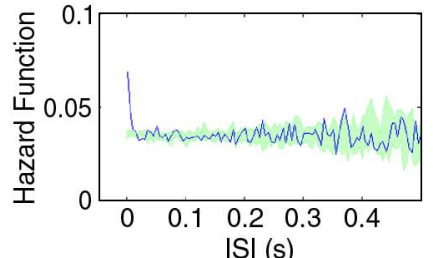

D

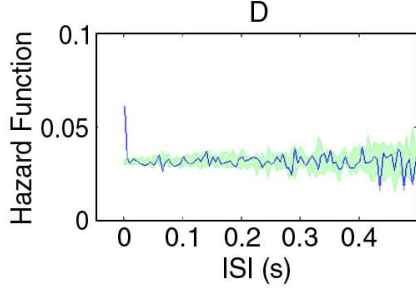

$\mathrm{F}$

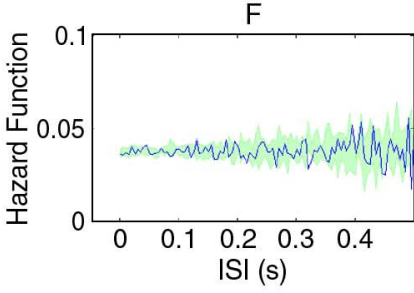

$\mathrm{H}$

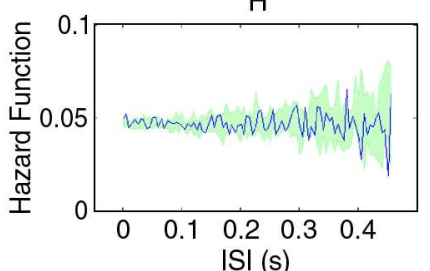

ISI (s)

Fig. 9. The spike trains for 8 SPADS (A-H) were recorded simultaneously. The blue line in each graph shows the hazard function of each spike train. The green envelope shows the range of hazard functions of the ISI between spike trains. The probability of firing remains the same; therefore, no correlations are present. The initial hazard function for SPADS B, C, D, and G are high due to the after-pulsing effect. This is an undesirable effect but can be removed by applying a refractory period to the SPAD [46].

SPADs (A-H). Each train was individually preprocessed to remove the effects of after-pulsing, and had its hazard function

calculated-the solid lines in Fig. 9. The ISI between all possible combinations of spike trains was also calculated and then the hazard functions generated. The worst case (max and $\min$ ) hazard function to a particular SPAD was calculated and is shown by the band. No high peaks in the hazard functions were detected, indicating that the spike trains can be regarded as independent. The curve fitting and coefficent of variation tests were performed for all the possible ISI combinations as further evidence, and the results are shown in Table I. The Adjusted $\mathrm{R}^{2}$ and $\mathrm{CV}$ for the spike trains recorded from the SPADs are shown in bold. The results for the ISIs between spike trains are all close to one, providing a high level of certainty of the spike trains' independence. The results for the SPAD generated spike trains $\mathrm{B}, \mathrm{C}, \mathrm{D}$, and $\mathrm{G}$ are further away from one. This is due to the after-pulsing effect not being entirely removed by the $200-\mu$ s preprocessing of the spike train. The effect of the after pulsing is clearly visible in Fig. 9, the probability of firing for SPAD B, C, D, and G is much higher just after the previous event. In a neural application, this does not pose a problem as a larger refractory period can, and will be, applied.

\section{Summary}

The three results presented in the previous section all underline that SPADs can produce Poisson-distributed spike trains, and that the spike trains from neighboring SPADs do not correlate.

The frequency of these spike trains can be controlled by the bias voltage VOP. Biologically plausible frequencies of up to $40 \mathrm{~Hz}$ can be achieved and, as each spike train is independent, they can be added together should faster frequencies be required.

\section{Probabilistic Cumulative Spike-Response Model}

This section introduces the proof-of-concept implementation of an avalanche diode-based neuron. An implementation of the model presented in Section II is evaluated through the attempted replication of the eight preclassified VMH cell behaviors. We show that seven of the eight behaviors are reproducible in addition to longer term behaviors such as spike adaptation and bursting. 


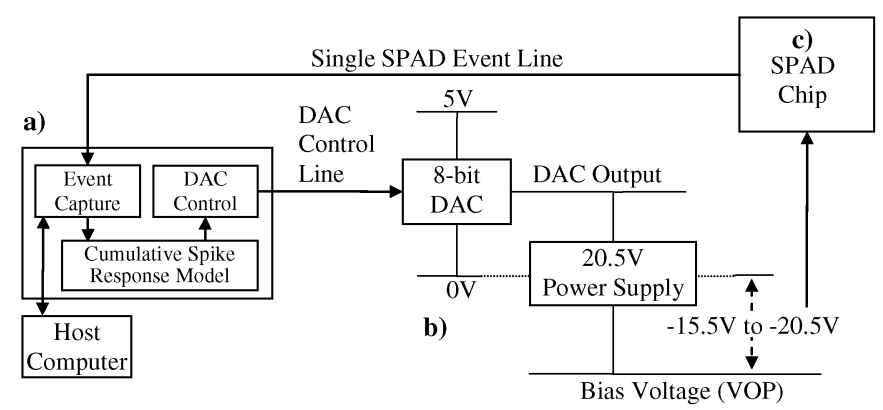

Fig. 10. Experimental setup. (a) An Opal Kelly FPGA board is connected by the universal serial bus to a PC, and configured with the ability to capture, timestamp, and stream SPAD events back to the PC, implement the CSRM, and send configuration data to the DAC every $1 \mathrm{~ms}$. (b) The negative bias voltage is generated by placing the fixed power supply between the DAC output (positive terminal) and the global VOP input to the SPAD IC (negative terminal). (c) The SPAD IC only has a single SPAD monitored.

\section{A. Implementation}

The neural model was implemented through the combination of a heavily light shielded SPAD and an FPGA-controlled 5-V 8-b digital-to-analog converter (DAC), see Fig. 10. This DAC was placed to modulate VOP between $20.5 \mathrm{~V}$ and $16.5 \mathrm{~V}$, the region that produces an almost linear relationship between mean dark count and VOP. This thereby allows the FPGA to linearly alter the probability of producing an event.

Within the FPGA, a state machine controls a cumulative spike-response (CSR) mechanism, which, in turn, controls the digital input to the VOP DAC. The model, which is a discrete implementation with a 1-ms time step, consists of three variables, each under a different constant decay, representing the HAP, DAP, and AHP of the neural model. If the SPAD produced one or more action potentials during the previous iteration ( $1 \mathrm{~ms}$ of time), a step increase or decrease is applied to each variable. These variables are combined with an offset variable to determine the final voltage produced by the DAC

$$
\mathrm{VOP}=\text { offset }+\mathrm{DAP}-\mathrm{HAP}-\mathrm{AHP} .
$$

The offset variable is used to position " 0 " so that the summed effects of the DAP or AHP will not cause clipping (See Fig. 11). The HAP is much larger than the DAP and AHP; hence, it is presumed that the HAP will clip. However, this is not a problem, as the clipping will force the SPAD into a region where avalanching is extremely unlikely to occur, hence enforcing a natural refractory period.

\section{B. Results}

The seven model control parameters (the magnitude and half life of the three decays, plus the offset) were tuned by hand to create a spike response that, when combined with the Poisson nature of the SPAD, would reproduce the likenesses of the patterns recorded from the VMH [35]. See Fig. 11 for an example of a generated spike response. The seven successful configurations are shown in Fig. 12, overlaid with their biological counterparts. The solid lines are the data generated by our model. The data recorded from the VMH are shown with a dashed line.

One of the recorded biological behaviors (regular firing) was found to be extremely difficult to recreate, with Fig. 13 showing

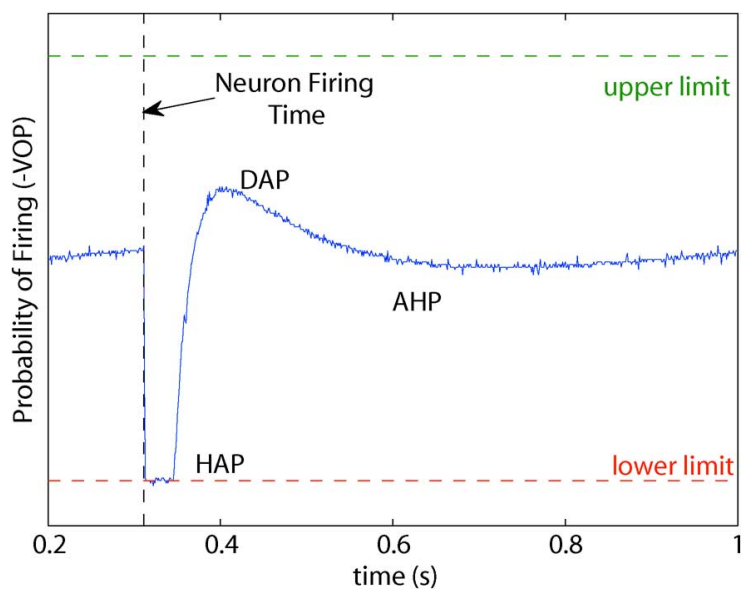

Fig. 11. Spike response pattern. This pattern, recorded from the circuit implementation, consists of the summation of three exponential decays-HAP, DAP, and AHP. The pattern is centered within the $5 \mathrm{~V}$ peak-to-peak region of the DAC by the offset parameter. Any value outside this region will be clipped by either the upper or lower limit.

the closest match found. While this trace has been confirmed as regular firing, it was only possible through the implementation of bursting behavior caused by DAP summation. This, in essence, created a composite non-homeostatic behavior, where the hazard is the combination of ISI distributions drawn from the model when it is in its elevated and suppressed states.

The cause of the model's inadequacy is rooted in the random nature of the SPAD's dark count, and how this is manipulated to produce different probabilities of firing. The probabilistic model uses the Poisson-distributed noise source to generate spike events, where the spike-response pattern is used to alter the mean firing rate of the noise source with time. This alters the probability of producing a spike event with respect to all preceding events. In this way, the spike response is a direct representation of the resulting hazard function. This also means that behaviors can only be produced if the shape of the hazard can be constructed from the three exponential decays. The hazard of the regular firing behavior consists of a refractory period, which dictates the mean firing rate, followed by a large DAP that falls sharply to a much lower probability of firing (determined by the base firing rate of the SPAD, see Fig. 13).

Initially, it was thought that this behavior could be created though the combination of two exponentials; a large negative going exponential to represent the refractory period, and a positive one to create a brief period of time directly after the refractory period that greatly elevates the probability of firing. However, because the model applies a step increase to all exponentials at the same time, the DAP must have a longer half life than the HAP to have a positive effect on the spike response. This poses several problems; First, it is not possible to create a sharp enough DAP decay while maintaining the refractory period. Second, since the half life of the DAP is greater than that of the HAP, and the model takes account of all previous activity, DAP summation can occur. This promotes a rudimentary bursting behavior, as shown in Fig. 13, where a self-sustaining plateau is formed that may randomly collapse and, as a result, longer interspike intervals can be generated randomly by the Poisson-distributed process. 


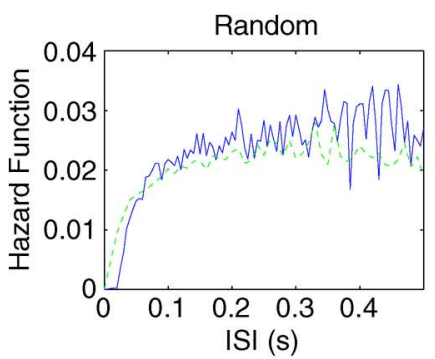

(a)

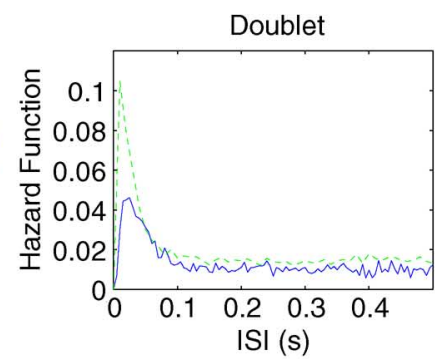

(b)

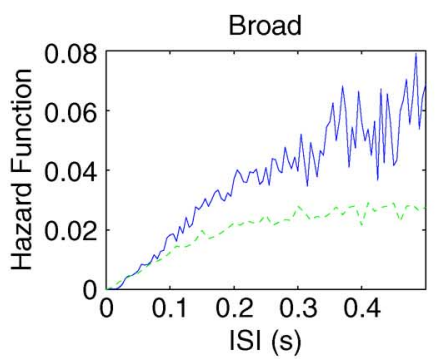

(c)



(d)

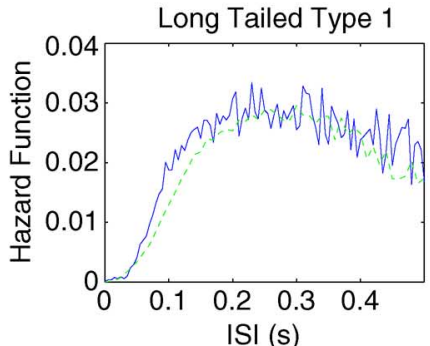

(e)

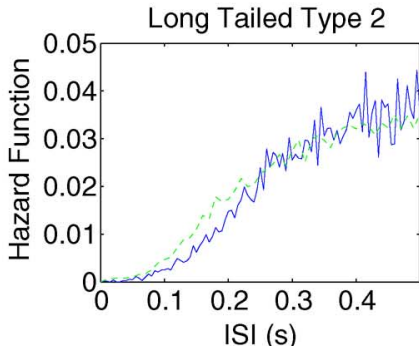

(f)

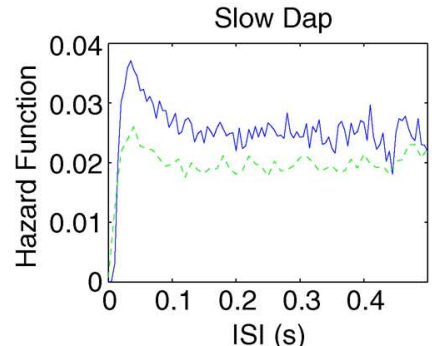

(g)

Fig. 12. Data from the SPAD SRM are the solid line. The dashed line is the VMH cell data. (a) Random behavior: characterized by a sharp HAP, to prevent immediate reactivation; and no further dynamics. (b) Doublet behavior: characterized by an extremely fast HAP followed by a sharp, but large DAP, to promote reactivation, and, hence, doublet behaviour. (c) Broad behavior: characterized by a slow but constant increase in probability of firing until a plateau is met. (d) Doublet broad behavior: characterized by a similar distribution to the broad behavior, but with a greatly increased chance of reactivation in the form of a doublet.

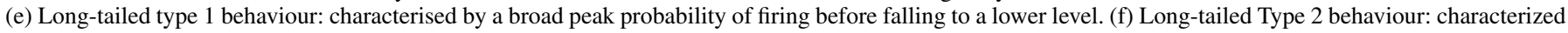
by the distinctive shape of the rise to constant probability of firing. This shape is caused by the combination of a low magnitude HAP and a shorter half life AHP (100 s of microseconds). (g) Slow DAP behavior: characterized by a small initial peak of activity before stabilizing at a constant probability of firing.

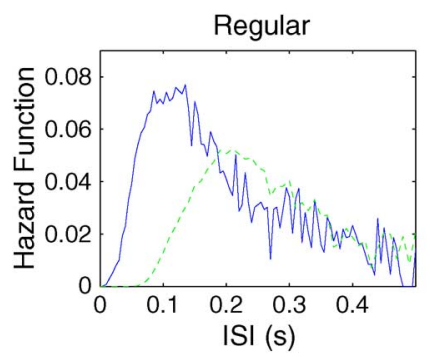

(a)

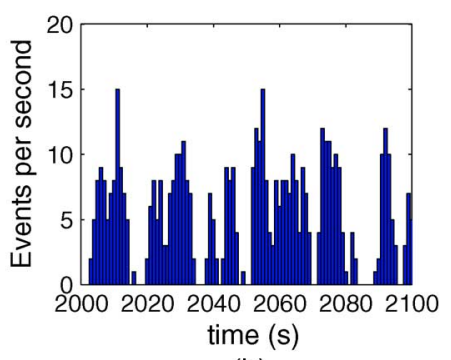

(b)
Fig. 13. (a) Regular behavior. The model allows that either the length of the refractory period or the decay rate after the peak activity be correct. This limitation means that the CSRM cannot truly recreate random firing activity. The data from the SPAD SRM are the solid line. The dashed line is the VMH cell data. (b) The spike rate was recorded from the SPAD SRM over a period of 100 $\mathrm{s}$ while attempting to recreate regular firing. Note the bursting behavior.

Ultimately, the recreation of regular firing behavior is impossible through the summation of exponentially decaying variables with synchronized step increases. However, if the correct spike-response pattern was created, then a true regular firing pattern could be recreated. This could be done by delaying the step increase of the DAP, thereby allowing it to have a shorter half life while still allowing it to have an effect on the resulting spike-response pattern, see Fig. 14.

\section{Spike Adaptation and Bursting}

Fig. 15 shows how the model can be made to produce bursting activity. This behavior is possible because the method used to construct the spike-response pattern, being cumulative, takes account of all previous activity. Therefore, the model can create

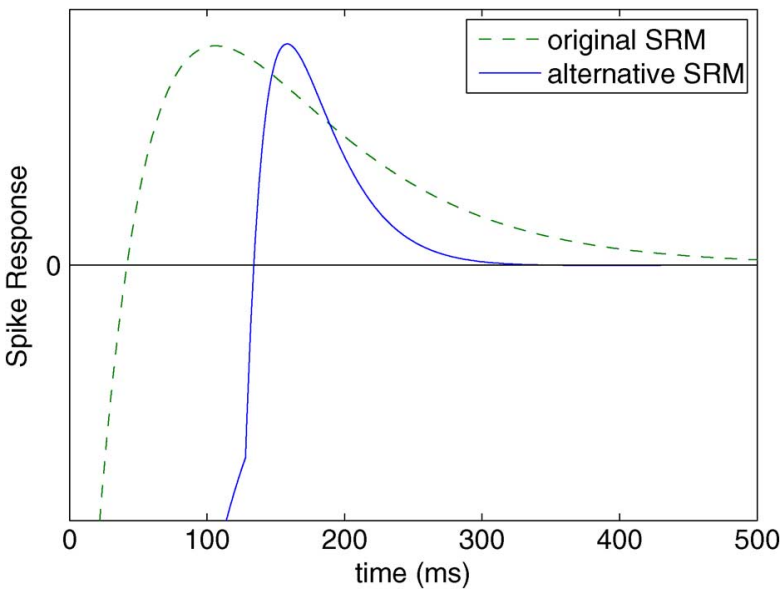

Fig. 14. Original and alternative SRM. In the alternative SRM, only the DAP is changed. Its half life is reduced and the step increase is only applied $100 \mathrm{~ms}$ after the spike event. The allows a long refractory period and a steep roll-off to be modelled.

longer term behaviors through the summation and interaction of the positive and negative going exponential decays.

In the case of spike adaptation, the longer term inhibitory effects of the AHP summate over multiple spike events, providing a negative feedback mechanism that naturally reduces the probability of firing, see Fig. 15 . The DAP can be configured to provide the opposite effect in the form of a short-term positive feedback mechanism. When combined with the longer term AHP, both of these effects interact to produce bursting, see Fig. 15. The DAP, having a larger magnitude, will accumulate more quickly, thereby further increasing the probability of firing. However, after a period of time, the AHP will become 


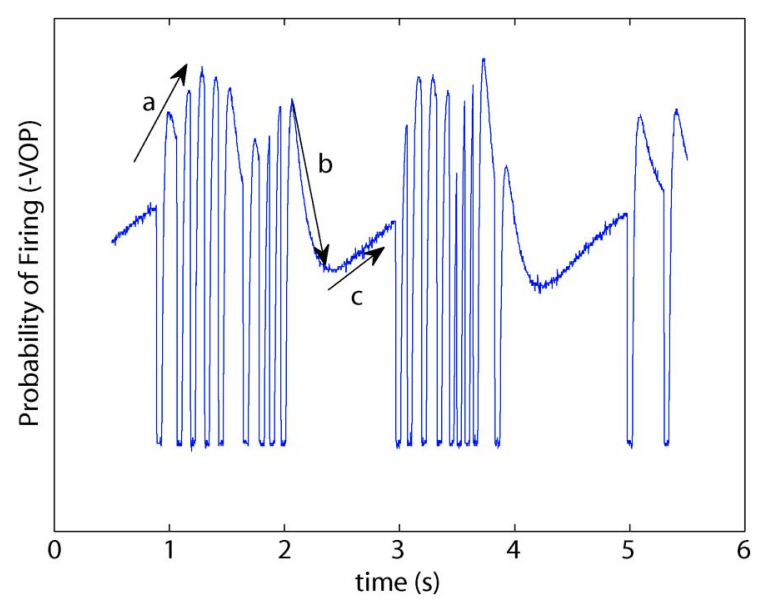

Fig. 15. Burst dynamics, recorded from the SPAD SRM, caused by interaction between the DAP and AHP. (a) The DAP accumulates rapidly, providing positive feedback. (b) The AHP magnitude becomes comparable with and, therefore, collapses the DAP plateau. (c) Period of silence while the AHP decays. Once this occurs, the cycle can repeat.

closer in magnitude and slow the firing rate to such a degree that the DAP summation will collapse. At this stage, the accumulated AHP remains, hence firing is halted until the AHP decays enough that the SPAD can fire again, and the cycle can repeat.

\section{Summary}

During this section, the Poisson-distributed SPAD output was modified with time by the inclusion of a CSR mechanism that consists of three exponential decays representing the HAP, DAP, and AHP intrinsic cell properties. This model was applied to the bias voltage and, therefore, altered the probability of firing with respect to previous spike events.

To evaluate this system, the model was adapted by hand to reproduce eight neural behaviors that were prerecorded from the VMH within the hypothalamus. Of these eight behaviors, seven were easily reproduced alongside spike adaptation and bursting, with only the regular firing behavior proving difficult to replicate.

This inadequacy was found to be caused by a limitation with the model, which cannot create a spike response with a sharper DAP decay rate than that of the HAP, which is responsible for enforcing the postfiring refractory period. This is not to say that this regular firing cannot be recreated; the hazard function of regular firing could be converted directly into a noncumulative SRM kernel that would naturally recreate this pattern. However, the removal of the cumulative nature of the CSRM would result in the inability to produce spike adaptation and bursting behavior.

\section{CONCLUSION}

This paper has introduced the SPAD device as a source of Poisson-distributed noise. We have shown that the dark count of an avalanche diode is: Poisson distributed; independent of other avalanche diodes in near proximity; and that the mean firing rate of the dark count is approximately linearly proportional to the magnitude of the negative bias voltage.

We then showed how this relationship can be utilized to implement a simple cumulative spike-response model with escape noise. This implementation represents a significant abstraction away from traditional models, where synaptic noise is integrated on a membrane, which then interacts with a deterministic firing mechanism. This is not to say that we could not implement such a model with SPAD noise sources; indeed, such a system could easily be constructed using multiple SPADs as independent synaptic inputs. However, since the long-term goal of this paper is to explore network activity on silicon, it is more practical to assume that the networks we aim to explore are fed by balanced tonic noise, and use a single device to represent the entire synaptic input and neuron.

Our model was tested and was capable of reproducing seven out of eight broad categories of single-cell spiking behaviors similar to those captured from the VMH region of the brain, as well as spike adaptation and bursting. We have explored the limitations of this model and show that regular firing activity is difficult to implement. This is, however, not a failure of the neural avalanche diode-based method, but rather a limitation of the model, which simply cannot produce a spike response of sufficient complexity to reproduce this regular behavior. The probability of firing for a given postfiring period of time can be derived empirically from the recorded interspike interval data in the form of hazard functions. This information can then be used to construct a more complex noncumulative spike-response kernel which could produce any desired behavior, including regular.

Although the work in this paper was motivated by the need for independent noise sources in a single-neuron spike-response model, the opportunities for avalanche-diode-generated noise are far wider. Models of spiking networks involving multiple noise sources will be made more compact, faster, and, thus, usable in the laboratory environment by the technology in this paper. Furthermore, noise-mediated effects, such as stochastic resonance [14], and noise-driven artificial neural networks, such as the Boltzmann machine [13], and its derivatives [47] need independent noise sources. If the promise of, for example, the continuous restricted Boltzmann machine as an embedded sensorfusion architecture [48] is to be fulfilled, multiple, integrated, compact, analog noise sources will be required. The SPAD-derived device described in this paper takes a fist step toward that hitherto-elusive goal.

\section{A. Future Work}

This work was carried out using a SPAD which was designed for low-light imaging tasks, such as florescent lifetime calculation [49]. The device has therefore been designed with photodetection in mind and, hence, has a large surface area to catch as many photons as possible. We do not require this detection. In fact, during this proof-of-concept study, the entire SPAD chip was heavily shielded from all light sources. Therefore, future work would first entail the design of an avalanching device designed specifically for our application. This device would be designed to have a large linear range of dark count rates to allow the more complex behaviors to be recreated. In addition, we would like to have a way of altering the probability of firing via a method that does not require rapid modification of a large negative voltage.

Ultimately, we would like to integrate the spike-response model, which is currently held within the FPGA and DAC, 
alongside the avalanche diode, to create an instantaneous probabilistic neural element that will allow the investigation of network behaviors with probabilistic systems, and provide a possible avenue for integration with biology.

\section{ACKNOWLEDGMENT}

The authors would like to thank K. Muir for his help with the test board and general understanding of the SPAD. Dave Laurenson suggested the method for proving the independence of the spike trains. The technical support of Europractice is gratefully acknowledged.

\section{REFERENCES}

[1] G. Indiveri, "A low-power adaptive integrate-and-fire neuron circuit," in Proc. IEEE Int. Symp. Circuits and Systems, May 2003, vol. IV, pp. $820-823$.

[2] A. Bofill-i-Petit and A. F. Murray, "Synchrony detection and amplification by silicon neurons with STDP synapses," IEEE Trans. Neural Netw., vol. 15, no. 5, pp. 1296-1304, Sep. 2004.

[3] M. Mahowald and R. Douglas, "A silicon neuron," Nature, vol. 354, pp. 515-518, 1991.

[4] J. Wijekoon and P. Dudek, "A CMOS circuit implementation of a spiking neuron with bursting and adaptation on a biological timescale," in Proc. IEEE Biomedical Circuits and Systems Conf., 2009, pp. 193-196.

[5] K. Rice, M. Bhuiyan, T. Taha, C. Vutsinas, and M. Smith, "FPGA implementation of Izhikevich spiking neural networks for character recognition," in Proc. Int. Conf. Reconfigurable Computing and FPGAs, 2009, pp. 451-456.

[6] S. Saïghi, Y. Bornat, J. Tomas, and S. Renaud, "Neuromimetic ICs and system for parameters extraction in biological neuron models," in Proc. IEEE Int. Symp. Circuits and Systems, May 2006, pp. 4207-4211.

[7] J. Schemmel, J. Fieres, and K. Meier, "Wafer-scale integration of analog neural networks," in Proc. IEEE Int. Joint Conf. World Congress on Computational Intelligence, 2008, pp. 431-438.

[8] J. M. Nageswaran, N. Dutt, J. L. Krichmar, A. Nicolau, and A. V. Veidenbaum, "A configurable simulation environment for the efficient simulation of large-scale spiking neural networks on graphics processors," Neural Netw., vol. 22, no. 5-6, pp. 791-800, 2009.

[9] D. F. M. Goodman and R. Brette, "Brian: A simulator for spiking neural networks in python," Frontiers Neuroinf., vol. 2, 2008.

[10] M. Djurfeldt, M. Lundqvist, C. Johansson, M. Rehn, O. Ekeberg, and A. Lansner, "Brain-scale simulation of the neocortex on the IBM Blue Gene/L supercomputer," IBM J. Res. Develop., vol. 52, no. 1, pp. 31-41, 2008.

[11] N. Brunel, "Dynamics of sparsely connected networks of excitatory and inhibitory spiking neurons," J. Comput. Neurosci., vol. 8, no. 3, pp. 183-208, May 2000.

[12] G. Leng, C. H. Brown, P. M. Bull, D. Brown, S. Scullion, J. Currie, R. E. Blackburn-Munro, J. Feng, T. Onaka, J. G. Verbalis, J. A. Russell, and M. Ludwig, "Responses of magnocellular neurons to osmotic stimulation involves coactivation of excitatory and inhibitory input: An experimental and theoretical analysis," J. Neurosci., vol. 21, no. 17, pp. 6967-6977, 2001

[13] G. E. Hinton and T. J. Sejnowski, "Learning and relearning in Boltzmann machines," in Parallel Distributed Processing: Explorations in the Microstructure Of Cognition. Cambridge, MA: MIT Press, 1986, vol. 1, Foundations, pp. 282-317.

[14] G. Deco and B. Schürmann, "Stochastic resonance in the mutual information between input and output spike trains of noisy central neurons," Phys. D, vol. 117, no. 1-4, pp. 276-282, 1998.

[15] S. Fusi, "Hebbian spike-driven synaptic plasticity for learning patterns of mean firing rates," Biol.Cybern., vol. 87, pp. 459-470, 2002.

[16] S. J. Upadhyaya, "Noise generators," in Wiley Encyclopedia of Electrical and Electronics Engineering, J. G. Webster, Ed. New York: Wiley, 1999, pp. 384-392.
[17] J. Alspector, B. Gupta, and R. B. Allen, "Performance of a stochastic learning microchip," in Advances in Neural Information Processing Systems. San Mateo, CA: Morgan Kaufmann, 1989, vol. 1, pp. 748-760.

[18] J. Alspector, J. Gannett, S. Haber, M. Parker, and R. Chu, "A VLSIefficient technique for generating multiple uncorrelated noise sources and its application to stochastic neural networks," IEEE Trans. Circuits Syst., vol. 38, no. 1, pp. 109-123, Jan. 1991.

[19] E. Chicca and S. Fusi, "Stochastic synaptic plasticity in deterministic aVLSI networks of spiking neurons," in Proc. World Congr. Neuroinf., 2001, pp. 468-477.

[20] W. Gerstner, R. Ritz, and J. L. van Hemmen, "Why spikes? Hebbian learning of time-resolved excitation patterns," Biol. Cybern., vol. 69, pp. 503-515, 1993.

[21] W. M. Kistler, W. Gerstner, and J. L. van Hemman, "Reduction of Hodgkin-Huxley equations to a threshold model," Neural Comput., vol. 9, pp. 1069-1100, 1997.

[22] R. Jolivet, T. J. Lewis, and G. W, "The spike response model: A framework to predict neuronal spike trains," in Proc. Int. Conf. Artifical Neural Networks and Neural Information Processing, 2003, vol. 2714 , pp. 846-853.

[23] W. Gerstner and W. Kistler, Spiking Neuron Models: Single Neurons, Populations, Plasticity. Cambridge, MA: Cambridge Univ. Press, 2002.

[24] W. Gerstner, "Spike-response model," Scholarpedia, vol. 3, no. 12, p. 1343,2008

[25] W. Gerstner, "Population dynamics of spiking neurons: Fast transients, asynchronous states and locking," Neural Comput., vol. 12, no. 1, pp. 43-89, 2000.

[26] R. Jolivet, A. Rauch, H.-R. Lüscher, and W. Gerstner, "Integrate-and-fire models with adaptation are good enough: Predicting spike times under random current injection," in Advances in Neural Information Processing Systems. : MIT Press, 2006, vol. 18, pp. 595-602.

[27] P. Roper, J. Callaway, and W. Armstrong, "Burst initiation and termination in phasic vasopressin cells of the rat supraoptic nucleus: A combined mathematical, electrical and calcium fluorescence study," $J$. Neurosci., vol. 24, no. 20, pp. 4818-4831, 2004.

[28] W. Gerstner, R. Kempter, J. L. van Hemmen, and H. Wagner, "A neuronal learning rule for sub-millisecond temporal coding," Nature, vol. 383, pp. 76-78, Sep. 5, 1996.

[29] R. Brette and W. Gerstner, "Adaptive exponential integrate-and-fire model as an effective description of neuronal activity," J. Neurophysiol., vol. 94, no. 5, pp. 3637-3642, 2005.

[30] E. Izhikevich, "Simple model of spiking neurons," IEEE Trans. Neural Netw., , vol. 14, no. 6, pp. 1569-1572, Nov. 2003.

[31] R. D. Andrew and F. E. Dudek, "Intrinsic inhibition in magnocellular neuroendocrine cells of rat hypothalamus," J. Physiol., vol. 353, pp. 171-185, 1984

[32] P. Roper, J. Callaway, T. Shevchenko, R. Teruyama, and W. Armstrong, "AHP's, HAP's and DAP's: How potassium curents regulate the excitability of rat supraoptic neurones," J. Comput. Neurosci., vol. 15, pp. 367-389, 2003.

[33] R. D. Luce, Response Times: Their Role in Inferring Elementary Mental Organization. Oxford, U.K.: Oxford Univ. Press, 1986.

[34] P. Janssen and M. N. Shadlen, "A representation of the hazard rate of elapsed time in macaque area LIP," Nature Neurosci., vol. 8, pp. 234-241, 2005.

[35] N. Sabatier and G. Leng, "Spontaneous discharge characteristic of neurons in the ventromedial nucleus of the rat hypothalamus in vivo," Eur. J. Neurosci., vol. 28, no. 4, pp. 693-706, 2008.

[36] R. Haitz, "Studies on optical coupling between silicon p-n junctions," Solid-State Electron., vol. 8, pp. 417-425, 1965.

[37] H. Finkelstein, M. J. Hsu, and S. C. Esener, "STI-bounded singlephoton avalanche diode in a deep-submicrometer CMOS technology," IEEE Electron Device Lett., vol. 27, no. 11, pp. 887-889, Nov. 2006.

[38] D. Stoppa, L. Pancheri, M. Scandiuzzo, L. Gonzo, G. F. Betta, and A. Simoni, "A CMOS 3-d imager based on single photon avalanche diode," IEEE Trans. Circuits Syst., vol. 54, no. 1, pp. 4-12, Jan. 2007.

[39] M. Gersbach, C. Niclass, E. Charbon, J. Richardson, R. Henderson, and L. Grant, "A single photon detector implemented in a $130 \mathrm{~nm}$ cmos imaging process," in Proc. 38th Eur. Solid-State Device Research Conf., Sep. 15-19, 2008, pp. 270-273. 
[40] M. A. Marwick and A. G. Andreou, "Single photon avalanche photodetector with integrated quenching fabricated in tsmc $0.18 \mu \mathrm{m} 1.8$ v CMOS process," Electron. Lett., vol. 44, no. 10, pp. 643-644, May 2008.

[41] A. Rochas, A. Pauchard, P. A. Besse, D. Pantic, Z. Prijic, and R. Popovic, "Low-noise silicon avalanche photodiodes fabricated in conventional CMOS technologies," IEEE Trans. Electron Devices, vol. 49, no. 3, pp. 387-394, Mar. 2002.

[42] S. M. Sze, Physics of Semiconductor Devices. New York: Wiley-Interscience, 1981.

[43] S. Cova, A. Lacaita, and G. Ripamonti, "Trapping phenomena in avalanche photodiodes on nanosecond scale," IEEE Electron Device Lett., vol. 12, no. 12, pp. 685-687, Dec. 1991.

[44] J. C. Jackson, D. Phelan, A. P. Morrison, R. M. Redfern, and A. Mathewson, "Toward integrated single-photon-counting microarrays," Opt. Eng., vol. 42, pp. 112-118, 2002.

[45] C. Niclass, M. Sergio, and E. Charbon, "A single photon avalanche diode array fabricated in deep-submicron CMOS technology," in Proc. Design, Automation and Test in Europe, Mar. 6-10, 2006, vol. 1, pp. $1-6$.

[46] K. Cameron, T. Clayton, B. Rae, A. Murray, R. Henderson, and E. Charbon, "Poisson distributed noise generation for spiking neural applications," in Proc. IEEE Int. Symp. Circuits and Systems, 2010, pp. $365-368$.

[47] H. Chen and A. Murray, "Continuous restricted Boltzmann machine with an implementable training algorithm," Proc. Inst. Elect. Eng., Vis., Image and Signal Process., vol. 150, no. 3, pp. 153-158, Jun. 2003.

[48] T. Tang, H. Chen, and A. Murray, "Adaptive, integrated sensor processing to compensate for drift and uncertainty: A stochastic 'neural' approach," Proc. Inst. Elect. Eng. Nanobiotechnology,, vol. 151, no. 1, pp. 28-34, Feb. 2004.

[49] B. R. Rae, K. R. Muir, Z. Gong, J. McKendry, J. M. Girkin, E. Gu, D. Renshaw, M. D. Dawson, and R. K. Henderson, "A cmos time-resolved fluorescence lifetime analysis micro-system," Sensors, vol. 9, no. 11, pp. 9255-9274, 2009.

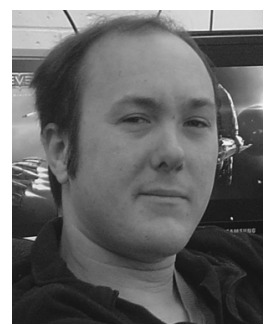

Thomas Clayton was born in 1981 in Surrey, U.K He received the M.Eng. degree in electrical and electronic engineering and the Ph.D. degree in electronic engineering and neuroscience from The University of Edinburgh, Edinburgh, U.K., in 2005 and 2009, respectively.

His $\mathrm{Ph} . \mathrm{D}$. project and current work integrates elements from electronic engineering, computer science, mathematics, and experimental and computational neuroscience, with his published works touching on graphics-processing unit and field-programmable gate-array-based hardware acceleration, evolutionary algorithms, probabilistic computational modeling, and exploration of the hippocampus and its interaction with the endocrine system. Currently, he holds research posts within the Centre for Integrative Physiology, the Institute for Perception, Action and Behavior, and the Institute for Integrated Micro and Nano Systems, all within the University of Edinburgh.

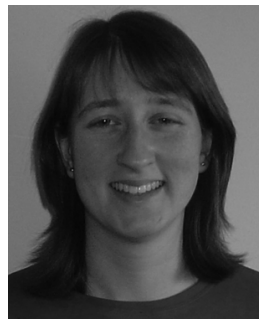

Katherine Cameron (S'04-M'06) was born in Edinburgh, U.K., in 1979. She received the M.Eng. degree (Hons.) in electronics and the Ph.D. degree in neuromorphic engineering from The University of Edinburgh, Edinburgh, in 2002 and 2007, respectively.

Currently, she is a Research Associate at the School of Engineering. Her current research interests include bioinspired engineering solutions to analog computation imperfections, mixed-signal very-large-scale integrated design, and neural computation.

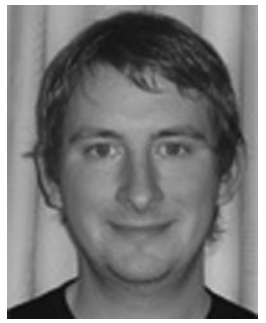

Bruce R. Rae (M'08) was born in 1983 in Aberdeen, U.K. He received the M.Eng. and Ph.D. degrees in electrical and electronic engineering from The University of Edinburgh, Edinburgh, U.K., in 2005 and 2009, respectively.

During his Masters degree, he was with ST Microelectronics Imaging Division. His Ph.D. project focused on the design and implementation of a lowcost, miniaturized complementary metal-oxide semiconductor (CMOS)-based microsystem for time-resolved fluorescence analysis. As of 2008, he was a Postdoctoral Research Associate at the Institute for Integrated Micro and Nano Systems which is part of the School of Engineering, The University of Edinburgh. His research interests include the design of CMOS-based systems for fluorescence lifetime analysis and single-photon counting and control circuitry for micro-light-emitting-diode devices.

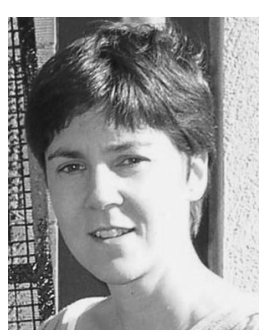

Nancy Sabatier received the M.Sc. degree in biochemistry and the Ph.D. degree in neuroendocrinology from the University of Montpellier, France.

She is a Research Fellow in the Centre for Integrative Physiology, the University of Edinburgh, Edinburgh, U.K. Her research focuses on the physiology of hypothalamic neurones, in particular, on their electrophysiological properties and their role in the regulation of feeding behavior. She is currently funded by a fellowship from Medical Research Scotland.

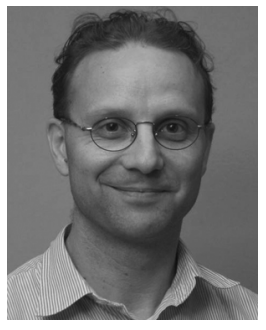

Edoardo Charbon (SM'11) received the M.S. degree in electrical engineering and electrical engineering and computer science from the University of California, San Diego, in 1991, and the Ph.D. degree in electrical engineering and electrical engineering and computer science from the University of California, Berkeley, in 1995.

From 1995 to 2000, he was with Cadence Design Systems and from 2000 to 2002, he was the Chief Architect of Canesta Inc. (now part of Microsft Corp.), where he developed high-speed image sensors. In 2002, he joined the Faculty of EPFL, where he founded the AQUA Group, a laboratory devoted to the study of complementary metal-oxide semiconductor quantum sensors for biophotonics and 3-D imaging. In 2008, he was appointed Professor at TU Delft, where he holds the Chair of VLSI Design. $\mathrm{He}$ has authored and coauthored many technical papers, 13 issued patents, and two books on very-large-scale integrated design, noise, and high-speed single-photon avalanche diode (SPAD) image sensors. His current research interests include 3-D sensing, biomedical imaging, and SPAD fundamentals.

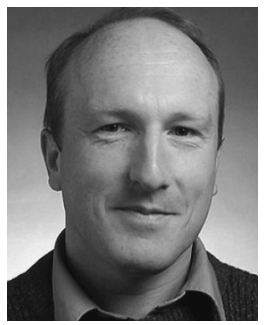

Robert K. Henderson (M'89) received the Ph.D. degree from the University of Glasgow, Glasgow, U.K., in 1990.

Currently, he is a Senior Lecturer at the School of Engineering in the Institute for Microelectronics and Nanosystems, University of Edinburgh, Edinburgh, U.K. Since 1991, he has been a Research Engineer at the Swiss Centre for Microelectronics, Neuchatel, Switzerland, working on low-power sigma-delta analog-to-digital converters and digital-to-analog converters for portable electronic systems. In 1996, he was appointed Senior VLSI Engineer at VLSI Vision Ltd., Edinburgh, where he worked on the world's first single-chip video camera and was Project Leader for many other complementary metal-oxide semiconductor (CMOS) image sensors. Since 2000, as Principal VLSI Engineer in the ST Microelectronics Imaging Division, he led the design of the first image sensors for mobile phones, resulting in annual revenues of several hundred million dollars. He joined the University of Edinburgh in 2005 to pursue his research interests in CMOS integrated-circuit design, imaging, and biosensors. As PI on the joint European project MegaFrame with three European Universities and ST Microelectronics, he has led research resulting in the first single-photon avalanche diode in nanometer CMOS technology. He is the author of many papers and 15 patents.

Dr. Henderson was awarded the Best Paper Award at the 1996 European Solid-State Circuits Conference as well as the 1990 IEE J. J. Thomson Premium. 


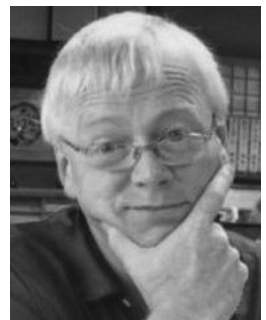

Gareth Leng received the B.Sc. degree in mathematics from the University of Warwick in 1974, and the M.Sc. degree in neurocommunications and the Ph.D. degree in physiology from the University of Birmingham, Birmingham, U.K., in 1975 and 1977, respectively.

He then was appointed Project Leader at the Babraham Institute, Cambridge, where he worked for 17 years. In 1994, he was appointed to the established Chair of Experimental Physiology at the University of Edinburgh, Edinburgh, U.K. Since 2007, he has been Head of the School of Biomedical Sciences, one of the four Schools that comprise the College of Medical and Veterinary Sciences at the university. His research interests span many areas of neuroendocrinology.

Dr. Leng is President of the International Neuroendocrine Federation.

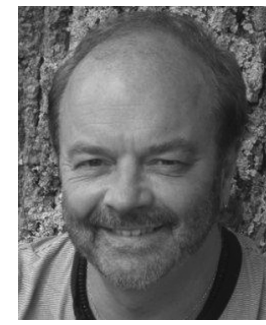

Alan Murray (M'91-SM'93-F'07) is Professor of Neural Electronics and Head of the School of Engineering at the University of Edinburgh, Edinburgh, U.K.

He introduced the pulse stream method for analog neural very-large-scale integrated circuits in 1985 . His interests are now in biologically inspired computational forms (particularly VLSI hardware), where noise and overt temporal behavior are important, and direct interaction between silicon and real neuronal cells and networks.

Prof. Murray is a Fellow of the Higher Education Academy, The Institution of Engineering and Technology, and the Royal Society of Edinburgh. He has published many academic papers. 